\title{
Automatic Detection of Display Defects for Smart Meters based on Deep Learning
}

\author{
Ye Chen ${ }^{1,2}$, Zhihu Hong ${ }^{1}$, Yaohua Liao ${ }^{1,2}$, Mengmeng Zhu' ${ }^{1}$, Tong Han ${ }^{1,2}$ \\ and Quan Shen ${ }^{3}$

\begin{abstract}
${ }^{1}$ Electric Power Research Institute of Yunnan Power Grid Co. Ltd, China
${ }^{2}$ Yunnan Key Laboratory of Green Energy, Electric Power Measurement Digitalization, Control and Protection, China

${ }^{3}$ Beijing University of Posts and Telecommunications, Beijing, China
\end{abstract}

\begin{abstract}
The smart meter is an essential part of an intelligent grid system. Defects in the LCD screen the smart meters affect their use. Therefore, detection of LCD screen defects of smart meters is of great significance for management and use of smart electricity meters. At present, detection methods are mainly realized by manual detection and automatic detection based on machine vision. However, performance of these two methods is not satisfactory. The fault detection task of a smart meter LCD screen can be divided into two parts: smart meter LCD localization and LCD fault detection. Therefore, this paper proposes a twostage system based on deep learning, which combines YOLOv5 with ResNet34. YOLOv5 is used for smart meter LCD localization and the classification network based on ResNet34 for LCD fault detection. We have constructed an LCD screen localization dataset and an LCD screen defect detection dataset to train and test our model. As a result, our model achieves a defect detection accuracy of $98.9 \%$ on the dataset proposed in this paper and can accurately detect the common defects of an LCD screen.
\end{abstract}

ACM CCS (2012) Classification: Networks $\rightarrow$ Network properties $\rightarrow$ Network structure

Keywords: smart meter, display defects, YOLOv5, ResNet34

\section{Introduction}

The defect detection for LCD screen is an important link in the whole life cycle quality certification of smart meters [1]. At present, defect detection in the LCD screen of smart meters is mainly carried out manually. However, it is un- reasonable to use the manual method for defect detection because of a large number of smart meters used in the power grid. Ruan Chunlei et al. [2] propose to use the template matching method to detect defects in LCD screens of smart meters. The method detects input images containing only the LCD screen region and achieves satisfactory results. However, the template matching method has poor robustness and is no longer applicable when the LCD screen size of the smart meter deviates from that of the template. On the other hand, it is challenging to accurately detect and position the LCD screen in natural scenes.

Currently, the research on LCD screen localization methods of smart meters mainly adapts LSD (Line Segment Detector) [3], [4] or Hough Transform [5]. The above method can achieve satisfactory localization accuracy for the LCD screen with complete appearance. However, when the target LCD screen has cracks as shown in Figure 1(a) or uneven illumination as shown in Figure 1(b), the identification accuracy of the model would be biased. At the same time, when the input image contains a large number of linear edge features, it is difficult to automatically filter the edge of the LCD screen through the LSD or Hough Transform methods, as shown in Figure 2.

At present, deep learning models have achieved excellent results in the research of feature ex- 
traction, target detection, and target recognition. This paper proposes using a deep learning model for the LCD screen localization and defect detection of smart meters. By extracting the features of the input smart meter images, the deep learning model can locate and detect defects of an LCD screen havingvarious-sized smart meter, while enhancing the robustness of the model and reducing the interference of external environmental factors on the model performance.

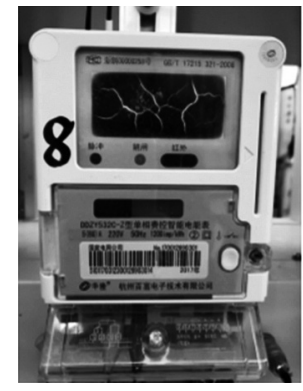

(a)

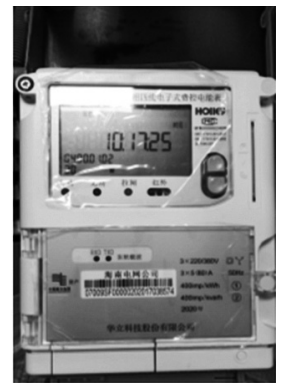

(b)
Figure 1. Samples of the LCD screen with cracks and uneven illumination.

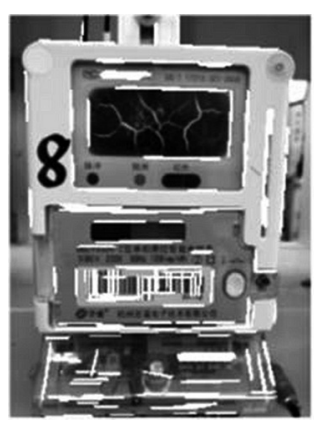

(a)

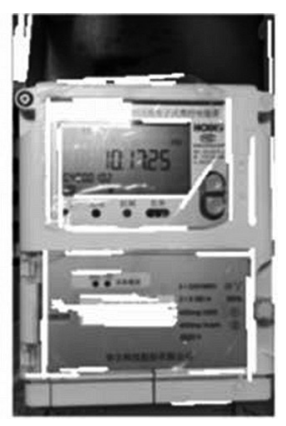

(b)
Figure 2. Detection result of the LSD and Hough Transform.

In summary, the contributions of our work are as follows:

1. We propose a method, which applies a deep learning model to localization and defect detection of smart meter LCD screens and establishes a two-stage defect detection model of smart meter LCD screens. The first stage is used to detect and extract the region of the LCD screen, and the second stage is designed to detect the defects of this region. We train and test the mod- el with the smart meter images taken in real scenes. The test results show that our model can achieve high accuracy in LCD screen localization and defect detection tasks.

2. We collect and make a defect detection dataset of smart meter LCD screens. The dataset is captured in a real LCD screen defect detection scene. Therefore, the model training with this dataset can be more convenient to deploy and use.

\section{Related Work}

At present, the research on the defect detection of smart meter LCD screen includes: LCD screen localization, LCD screen defect detection, and the combination of the localization and the defect detection steps for LCD screens. We introduce the existing work from the following three aspects: LCD screen localization, LCD screen defect detection, and the two-stage model that combines localization with defect detection.

\subsection{LCD Screen Localization}

Ruan Chunlei et al. [2] propose a method, which uses the gray map, Gaussian filtering, and binarization algorithm to preprocess the input image. Then, they use the contour detection algorithm based on OpenCV to get the contour sequence of the preprocessed image. Using the LCD screen features, such as the area and the number of corner points, they get the contour of the LCD screen. In the final step, the model detects the display defects of the LCD screen through a differential operation between the input LCD screen image and the template image.

Shi Kang et al. [6] use the geometric matching method based on LabVIEW to calculate the center position of the LCD screen directly. They crop the input image by using the center position, length, and width of the LCD screen. Finally, they compare the difference between the cropped LCD screen image and the LCD screen template to detect display defects.

Zhang Zhao et al. [7] use median filtering and graying to preprocess the input image. They use the Prewitt operator and Sobel operator to ex- 
tract the edge in the preprocessed image. And then use the dilation and erosion operations to get the largest connected area in the image, which is the LCD screen. After that, by using horizontal projection and vertical projection, the display characters of the LCD screen are segmented. Finally, they use the square difference matching algorithm to detect display defects of the LCD screen.

\subsection{LCD Screen Defect Detection}

At present, the LCD defect detection method is greatly affected by illumination. To solve this problem, QIAO W Y et al. [8] improve the LBP algorithm. They use the improved LBP algorithm to binarize the LCD screen images of the smart meter. The improved LBP algorithm has a better binarization performance than the original LBP algorithm or other binarization algorithms if the image of the LCD screen is captured under strong or weak lighting conditions. Overall, the better binarization results of the LCD screen images improve the defect detection accuracy of the smart meter LCD screen.

Song R et al. [9] propose a self-learning fast template matching method based on the sequential similarity detection algorithm (SSDA). For the sequential similarity detection algorithm, they use an adaptive threshold to replace the fixed threshold. The improved algorithm can reduce the parameters of calculations on non-matching points and achieves a faster detection speed.

Anis A et al. [10] use the characteristics of color light emitted by the LCD screen of smart electricity meters. Therefore, they convert RGB input images to $\mathrm{YCbCr}$ images from which the LCD screen can be easily cropped. The Canny operator is then used to extract the edges, and the displayed characters are segmented using horizontal and vertical pattern features. In the final step, they identify the display sequence of the LCD screen.

Quintanilha D et al. [11] transform the input image from the RGB to HSV. And then, they use the LCD screen templates to identify and segment the LCD screen. They use the Histogram of Oriented Gradients (HOG) to get the segmented images of the display digits. In the last step, they use the SVM algorithm to recognize the images.

\subsection{LCD Screen Localization and Defect Detection}

Wang Shujing et al. [12] use back-projection of RGB image histogram and binarization to preprocess the input image. Then they use the Hough transform to extract the edge line of the LCD screen. They remove the background in the LCD screen images by binarizing the extracted LCD screen images. The display characters of the extracted LCD screen are divided through horizontal and vertical projection. Finally, the square difference matching algorithm is used to match the template of the displayed characters with the divided display characters to judge the display defects.

Peng Yu et al. [13] use the weighted average gray algorithm to transform the input images to grayscale. Then, they use the line segment detector (LSD) algorithm to detect the line above the LCD screen. With the results of LSD, they correct the tilt angle of the input image. Then, they use the correlation coefficient matching algorithm to achieve the localization and segmentation of the LCD screen, and the single character images of the LCD screen can be obtained. Finally, they use a convolutional neural network to recognize every single character and detect the display defects.

Pei B et al. [14] proposed to use the LSD algorithm to correct the tilt of the input image. The LCD screen is separated into the ordinary area and the digital tube area. They use a convolutional neural network $(\mathrm{CNN})$ to locate and identify the ordinary character. The template matching algorithm is used to determine the digital character in the digital tube area. And this method gets state-of-the-art performance.

Salomon G et al. [15] realize automatic meter reading through Fast RCNN [16] and YOLO (You Only Look Once) [17] algorithm. Fast RCNN and YOLO are used to directly locate and identify smart electricity meters' dial and realize automatic data reading of smart meters. Most importantly, for smart meter display area localization, their method achieves $83 \%$ IOU (Intersection Over Union) accuracy, showing excellent performance. 


\section{Datasets}

At present, there is no suitable dataset for the task of smart meters LCD screen localization. Therefore, we construct the LCD screen localization dataset in order to train and test the LCD screen localization model. All the images of this dataset are collected from smart meter sorting factories. At the same time, we construct the LCD defect detection dataset to train and test the LCD defect detection model.

\subsection{LCD Screen Localization Dataset}

In the process of smart meter production and testing, the smart meters are fixed on the movable terminals which are on the production line. Therefore, the attitude of the smart meters in the camera's view remains constant when we capture the images. By adjusting the relative position of the camera and the electricity meters, we ensure the electricity meters stay with a horizontal attitude in the camera's vision. On the premise, we use a horizontal rectangular box to delineate the region of the smart meter LCD in an image. The box is represented by $(X, Y, W, H)$, where $(X, Y)$ is the coordinate of the center point of the rectangular box, and $(W$, $H)$ is the width and height of the rectangular box. We visualized the labeled data samples, as shown in Figure 3. The horizontal rectangular box in the sample image shows the position of the LCD screen we labeled.
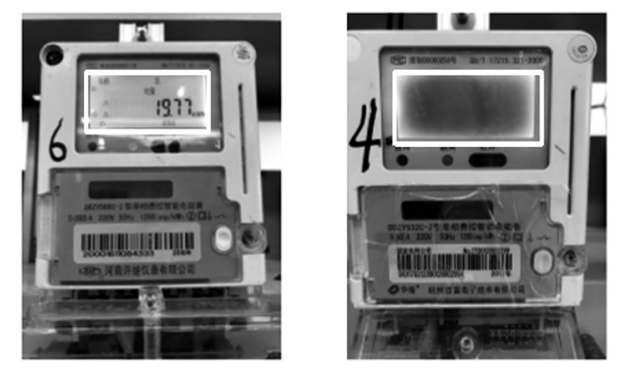

Figure 3. Samples of the LCD screen localization dataset.

\subsection{LCD Screen Defect Detection Dataset}

We construct the LCD screen defect detection dataset by manually cropping the parts containing LCD screen regions from the smart meter images. Using the smart meter images we col- lected, we classified the LCD screens into four types:
a) normal display,
b) no display,
c) incomplete display,
d) abnormal display, and
e) liquid crystal rupture, as is shown in Fig- ure 4.

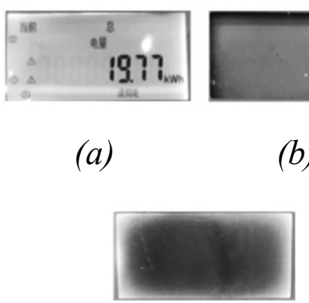

(d)

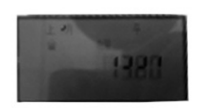

(c)

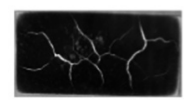

(e)
Figure 4. Samples of the LCD screen defect detection dataset.

We divide this dataset into two parts, the training set and the testing set for the LCD screen localization dataset. The training set has 357 samples, including 125 samples with normal display, 98 samples with no display defect, 80 samples with abnormal display defect, 28 samples with liquid crystal rupture defect, and 26 samples with incomplete display defect. The test set has 152 samples, including 53 samples with normal display, 42 samples with no display defect, 34 samples with abnormal display defect, 12 samples with liquid crystal rupture defect, and 11 samples with incomplete display defect. An XML file labels the LCD screen position of the samples in the dataset. Thus, models can obtain the location of the LCD screen by searching the XML file. The images of the dataset are captured by a mobile phone camera. There are two kinds of image resolution in the dataset, $1080 \times 1440$ and $3024 \times 4032$.

We use the same sample partition ratio for the LCD defect detection dataset as the LCD localization dataset. However, the sample images of this dataset are the manually cropped LCD screens, and the class information of the corresponding LCD screen has also been saved in an XML file. All the datasets are available by contacting the corresponding author. 


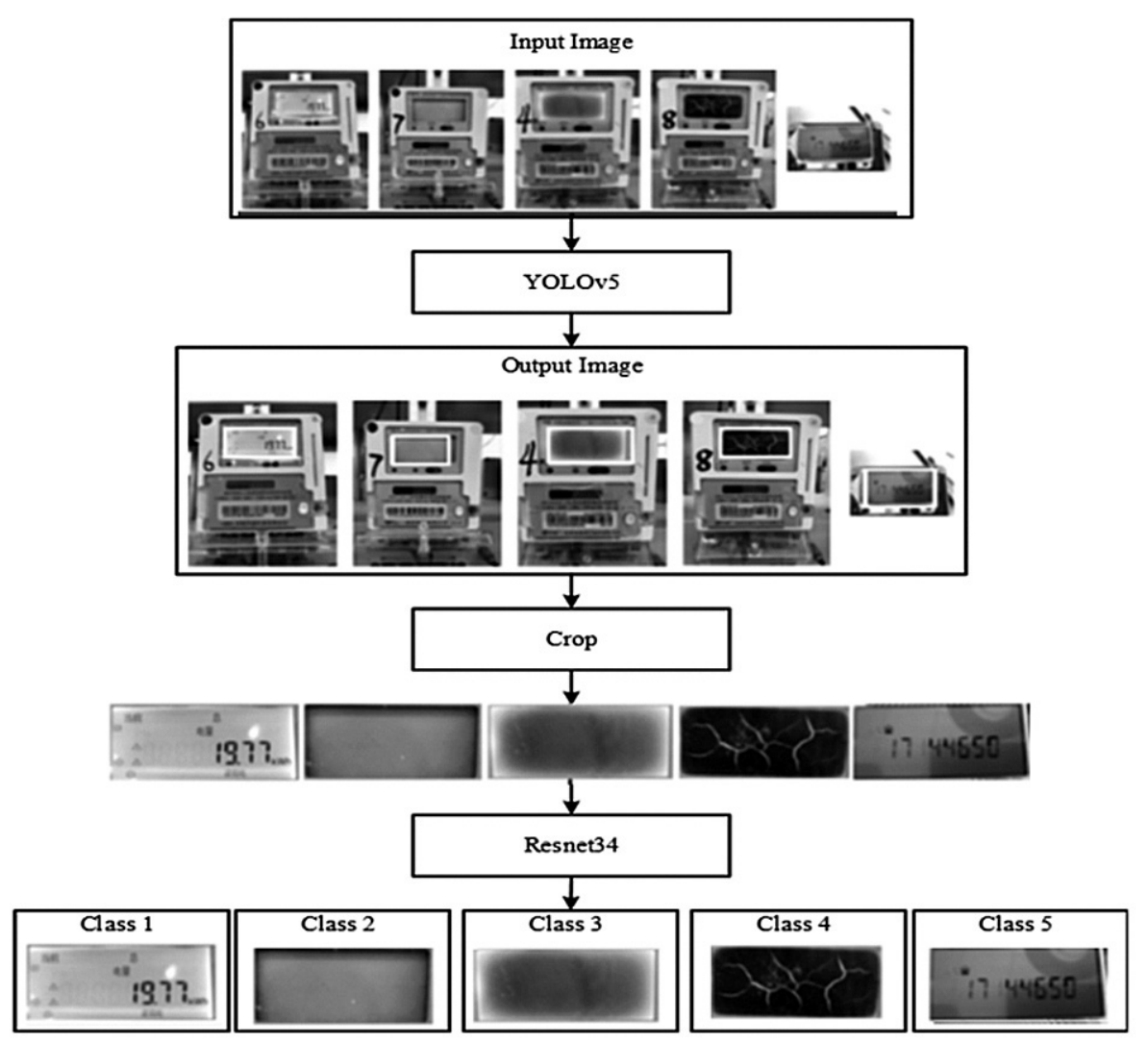

Figure 5. Architecture of the two-stage LCD defect detection model.

\section{Network Architecture}

This paper combines the LCD screen localization network with the LCD screen defect detection network and proposes a two-stage LCD screen defect detection model for smart meters. The overall structure of the model is shown in Figure 5. In the first stage, the model takes the image that contains the smart meter and background information as input to predict the position of the LCD screen in the image. And then, the model crops the LCD screen with the predicted position. In the second stage, the model feeds the cropped images into the LCD screen defect detection model to predict the defect type.

In this section, the detailed introduction of the model is divided into two parts: 4.1. LCD screen localization model and 4.2. LCD screen defect detection model.

\subsection{LCD Screen Localization Model}

The pictures of smart meters we collected contain a lot of information unrelated to the LCD screen, such as the area outside the LCD screen of the smart meter and the background, which has no use to detect LCD screen defects. Moreover, it costs more time to detect defects with the whole image. Therefore, the LCD screen needs to be localized first. And the following defect detection model will focus on the display area obtained by localization to avoid irrelevant information and reduce the detection time.

To position the LCD screen, we use the YOLOv5 algorithm, which contains classification and location functions in a one-stage model. Also, the YOLOv5 achieves a high speed in the task of target detection. With this advantage, the YOLO series model [17], [18], [19], [20] is widely used in target detection.

The structure of the YOLOv5 network is shown in Figure 6, which can be divided into three parts: Backbone, Neck, and Head. YOLOv5 selects CSPDarkNet [21] (Cross Stage Partial Networks) as the Backbone part, extracting rich features from input images. The Neck part generates a feature pyramid based on the feature map extracted from the Backbone part. The 
feature pyramid can enhance the recognition ability of the model for different sizes and scale targets [22]. At the same time, the Neck part fuses the feature maps of different scales. Because, in the process of feature extraction of the Backbone part, the non-significant features in the feature maps will be lost, which will affect the model's performance. By fusing the feature maps, the non-significant feature loss caused by the down-sampling operation can be effectively avoided [23],[24]. In the Neck part, the feature maps of different scales are fused by up-sampling and concatenation, and finally, the fused feature maps of $19 \times 19,38 \times 38$, and $76 \times 76$ are obtained. The primary function of the Head is to position the LCD screen. The Head applies three specific anchor sizes to feature maps and predicts category probabilities, scores, and offsets for each anchor frame. The Head has three different scales to detect objects of various sizes, and each outputs a tensor with 255 channels.

The loss function of the YOLOv5 network contains three parts: objectness score loss (category loss), class probability score loss (confidence loss), and the regression loss of the bounding
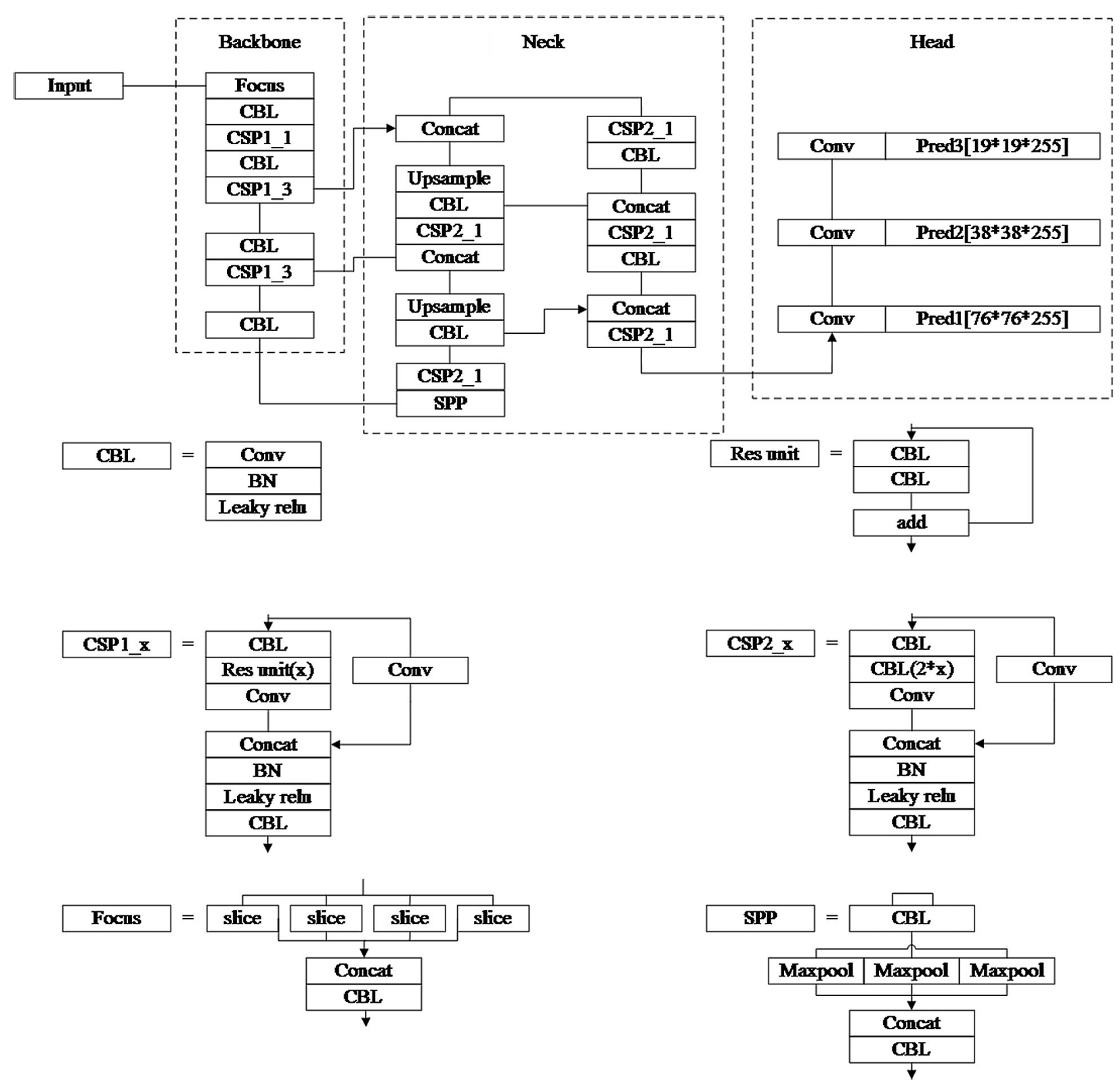

Figure 6. Architecture of the YOLOv5 model. 
box. The category loss is calculated by Binary Cross Entropy with Logits loss function as shown in formula (1).

$$
\begin{aligned}
& \operatorname{Loss}_{\text {BCELogits }}= \\
& =-\frac{1}{n} \sum_{i=1}^{n}\left[y_{i} \log \sigma\left(x_{i}\right)+\left(1-y_{i}\right) \log \sigma\left(1-x_{i}\right)\right]
\end{aligned}
$$

Where $y_{i}$ is the target value of categories and $x_{i}$ is the prediction category of the model. $\sigma()$ means the sigmoid operation.

The confidence loss is calculated by the Binary Cross Entropy function as shown in formula (2).

$$
\begin{aligned}
& \operatorname{Loss}_{B C E}= \\
& =-\frac{1}{n} \sum_{i=0}^{n}\left[y_{i} \log \left(x_{i}\right)+\left(1-y_{i}\right) \log \left(1-x_{i}\right)\right]
\end{aligned}
$$

Where $y_{i}$ is the value of 0 or 1 . While the category of the prediction is the same as the target category then $y_{i}$ equals 1 . If the prediction category is different from the target category then $y_{i}$ equals 0 . The $x_{i}$ is confidence value of the model to the prediction.

The YOLOv5 selects GIOU_Loss [25] as the loss function of the bounding box. The formula is as follows:

$$
\begin{gathered}
G I o U=I o U-\frac{\left|A_{c}-U\right|}{\left|A_{c}\right|} \\
\operatorname{Loss}_{G I o U}=1-G I o U
\end{gathered}
$$

Where $I o U$ is the ratio of intersection to the union between the predicted bounding box and ground truth of the LCD screen position, $U$ is the area of the union between the predicted bounding box and ground truth of the LCD screen position. $A_{C}$ is the area of the minimum enclosing rectangle between the predicted bounding box and ground truth of the LCD screen position.

\subsection{LCD Screen Defect Detection Model}

LCD screen defect detection model uses ResNet34 as the backbone to extract the features of the LCD screen. ResNet is a deep convolution neural network model constructed by residual mechanism [26]. There are two branches for the residual module: one is composed of two convolution layers, and the other is shortcut connections. The shortcut connection transfers the input of the residual module directly to the output as the initial result of the output. In the most extreme case, the convolution layer learning rate of the residual module is 0 . The residual module can ensure that the output is the same as the input, rather than 0 . Therefore, the residual structure can avoid the gradient disappearance and gradient explosion caused by the increase of network depth and realize effective training of the deep neural network. The ResNet34 backbone network extracts the features from the input images and generates feature maps with channel dimension 512. After average pooling, the feature maps are entered into the fully connected layer to detect and classify defect categories. The fully connected layer outputs a vector containing $n$ elements, each representing the predicted value for each type of label. The overall structure of the defect detection network is shown in Figure 7.

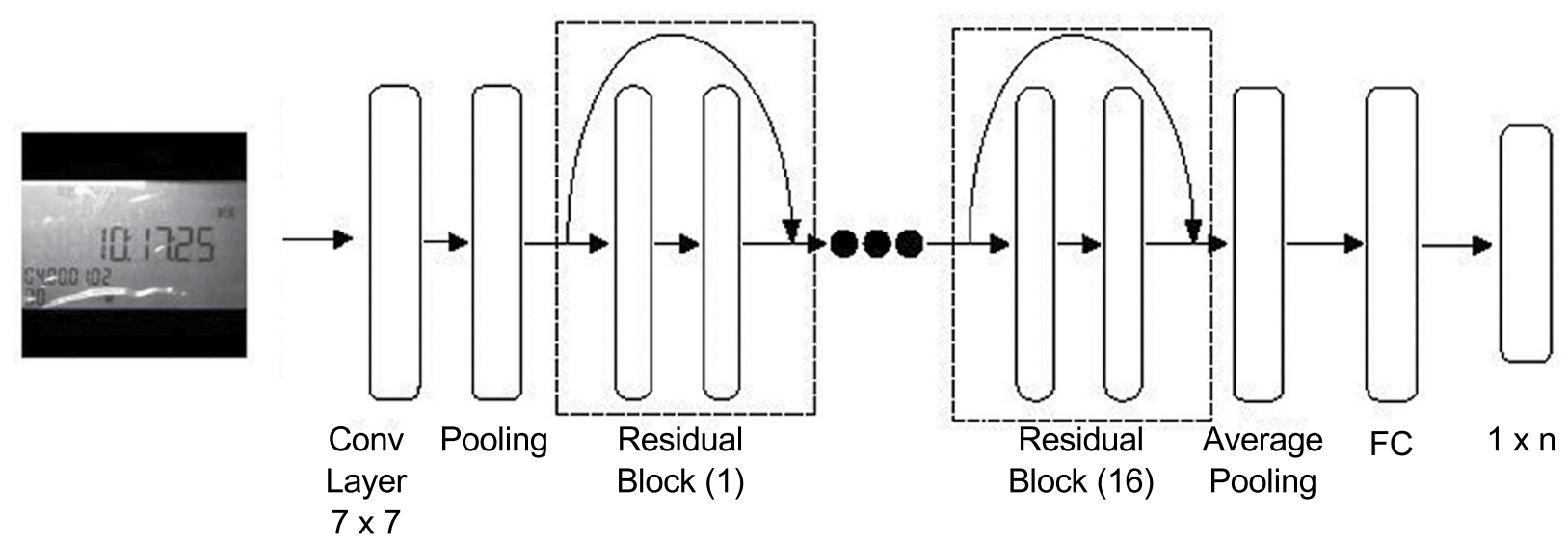

Figure 7. Architecture of the ResNet34 model. 
The ResNet34 model uses cross-entropy as the loss function and its calculation formula is as follows:

$$
C E(p, q)=-\sum_{i=1}^{C} p_{i} \log \left(q_{i}\right)
$$

Where $C$ is the number of the defect types, $p_{i}$ is the ground truth of the category label for the input image, $q_{i}$ is the predicted value of the LCD screen defect detection model.

\section{Experiment}

This section introduces the experiment from three aspects: training details of LCD screen localization model, training details of LCD screen defect detection model, and evaluation.

\subsection{Training Details of LCD Screen Localization Model}

The YOLOv5 model enhances the training data by cutting, scaling, and splicing. The number of training samples has increased five times. With the data augmentation, the performance of the YOLOv5 gets improved obviously. We visualized the data augmentation, and the result is shown in Figure 8. The training samples with data augmentation in Figure 8 contain several original training samples and some blank areas. Compared with the original training samples, the training sample after data augmentation includes multiple LCD screens. At the same time, the size and position of the LCD screen are more variable.
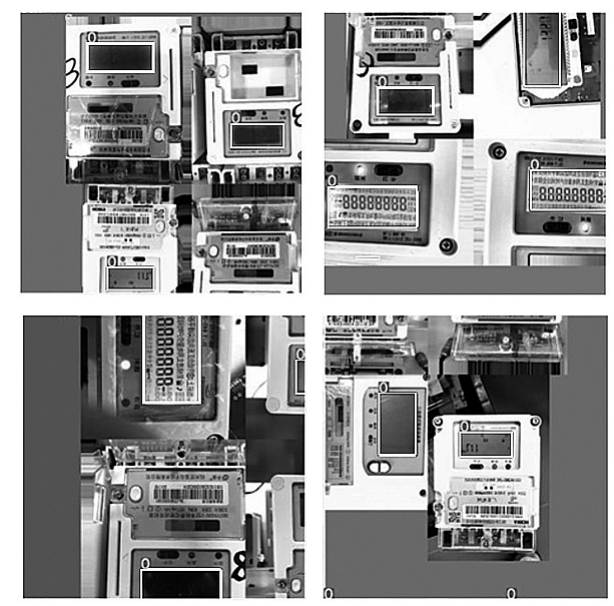

Figure 8. Sample of the data augmentation.
In this paper, we choose Adam as the optimizer of the YOLOv5 network and other parameters are set as follows. We use a batch size of 10 and run the algorithm for 100 epochs, with weight decay set to 0.0005 , and learning rate set to 0.001 . Results of the localization model in the training process are shown in Figure 9. The model's training loss and verification loss converge slowly in the initial stage but rapidly after 20 steps. After training for 100 epochs, both the training loss and verification loss converge to a low value, and the performance of the model tends to be stable. The recall and average accuracy fluctuate significantly in the initial training stage but increase rapidly after 20 steps. After model is trained for 100 iterations, the recall rate is close to 1 , and the mAP50 of the model exceeds $95 \%$.

\subsection{Training Details of LCD Screen Defect Detection Model}

In this paper, we choose Adam as the optimizer of ResNet34. For the training of ResNet34, we used a learning rate decay of 0.0002 , with a batch size of 12 and an epoch setting to 100 . The loss value and accuracy of the model in the training process are shown in Figure 10. In the initial stage, the loss decreases rapidly, and the accuracy increases quickly. In the middle and the end of the model training process, the loss tends to wavelike converge, and the accuracy tends to wavelike rising.

\subsection{Evaluation}

It is challenging to make a fair performance comparison with the previous methods [2], [14]. Because of the development of hardware computing capability and DNN technology, it is difficult to determine how much the method proposed by us contributes to LCD localization and defect detection performance improvement.

In this paper, we not only compare the accuracy of our model with the accuracy that the previous methods announced, but also we replicate the previous method with our equipment by our dataset. By comparing the results with the replicate results, we can judge the superiority of different models, and we can achieve a fairer comparison result. 


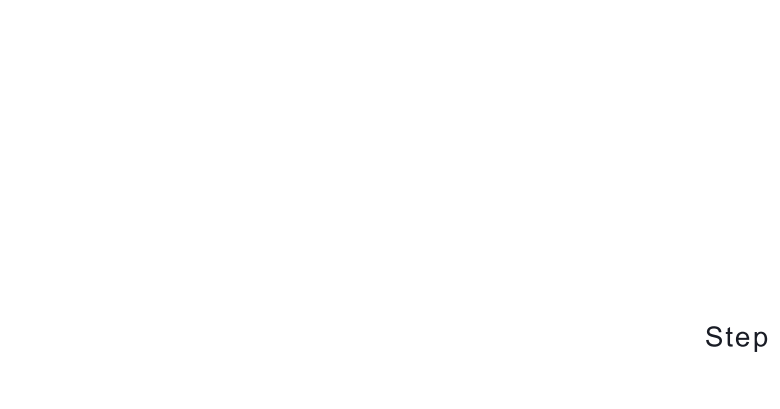

(a)

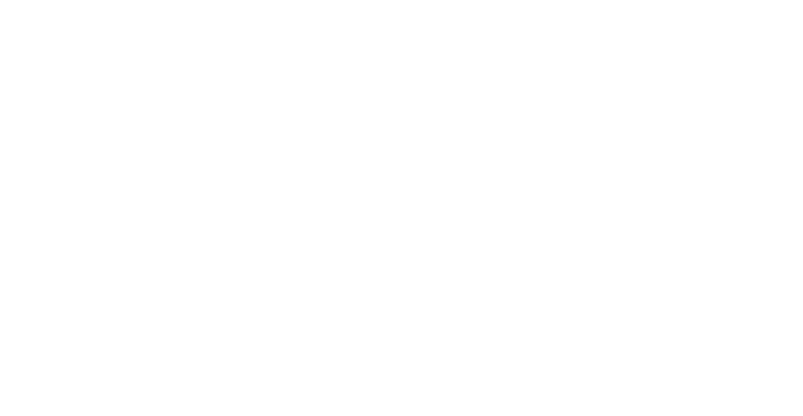

(c)

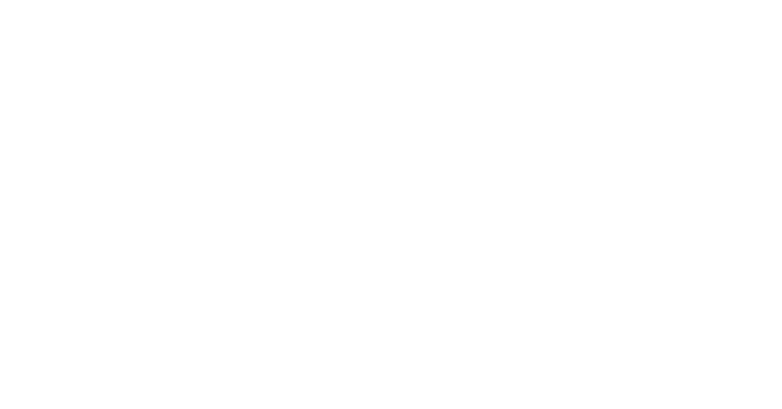

(b)

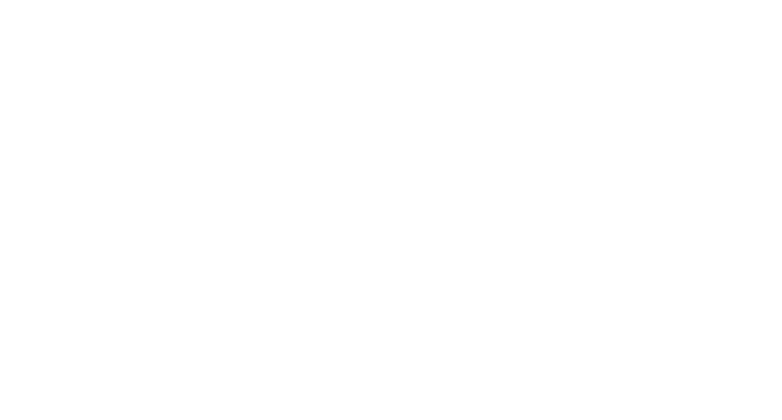

(d)

Figure 9. Training process of the YOLOv5 in different epochs.

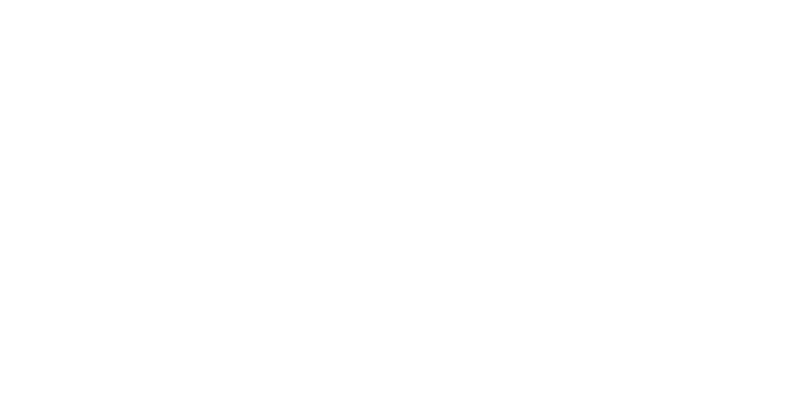

(a)

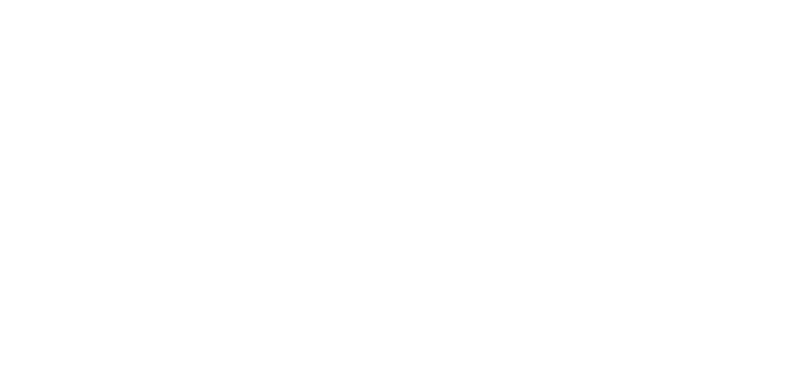

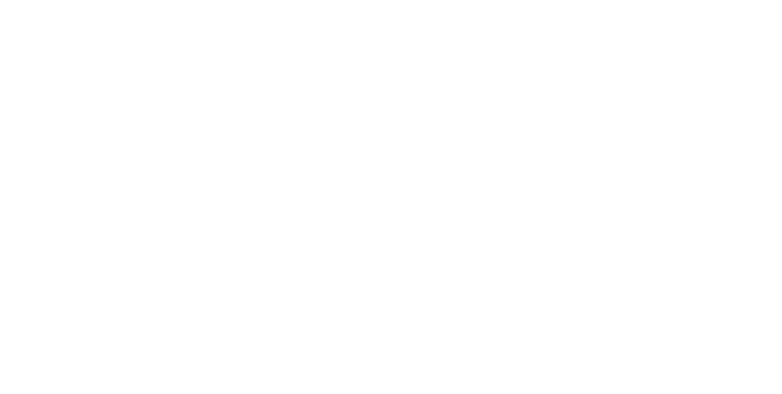

(b)

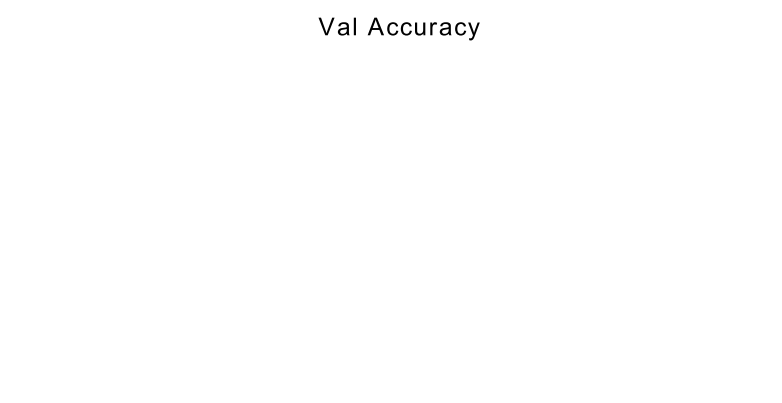

(d)

Figure 10. Training process of the ResNet34 in different epochs. 


\subsubsection{Evaluation of The LCD Screen Localization Model}

The result of the basic global thresholding method and our method is shown in Figure 11. Figure 11(a) is the preliminary detection result of the LCD screen contour by the basic global thresholding method, including many candidate bounding boxes. Figure 11(b) shows the contour of the LCD screen after manual selection. Due to the limitations of the basic global thresholding method, even manually selected methods can not accurately detect the entire contour of the LCD screen. As shown in the second and third samples in Figure 11(b), the LCD screens are not entirely detected due to LCD display defects and uneven illumination of the shooting. Figure 11(d) is the detection result of the partial image by the basic global thresholding method. Few candidate boxes are generated in case of partial detection, it is easy to select the contour of the LCD screen, but incomplete detection still exists.

According to the above experimental result, we can find out that the basic global thresholding method is greatly influenced by the resolution of the smart meter image, the image shooting angle, and the environmental illumination. When the environmental illumination is not uniform, the LCD screen defects are present or the background is cluttered, the basic global thresholding method cannot accurately localize the position of the LCD screen.

We use YOLOv5 as the LCD localization model, which uses deep convolutional networks to extract the features of input images from different scales. The anti-interference ability of our model towards the external environment and the robustness of our model is improved. The LCD localization results of the YOLOv5 model are shown in Figure 11(c). The model can still accurately detect the position of the LCD screen in the case of uneven illumination or display defects.

The results show that the YOLOv5 has stronger robustness than the basic global thresholding method in localization of LCD screens. The YOLOv5 network can detect more complex LCD screens and can be used in a changeable detection environment.

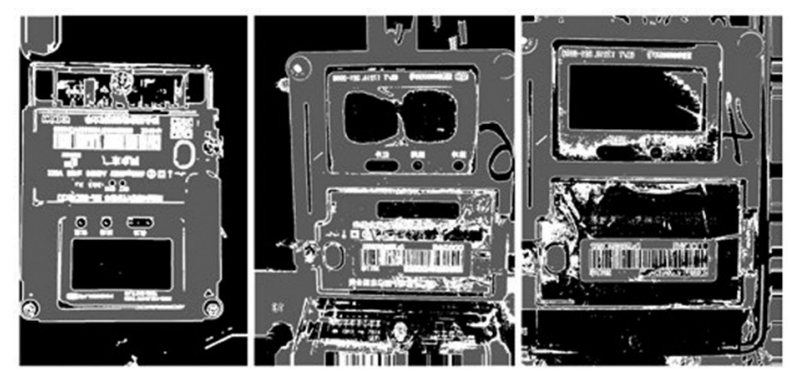

(a)
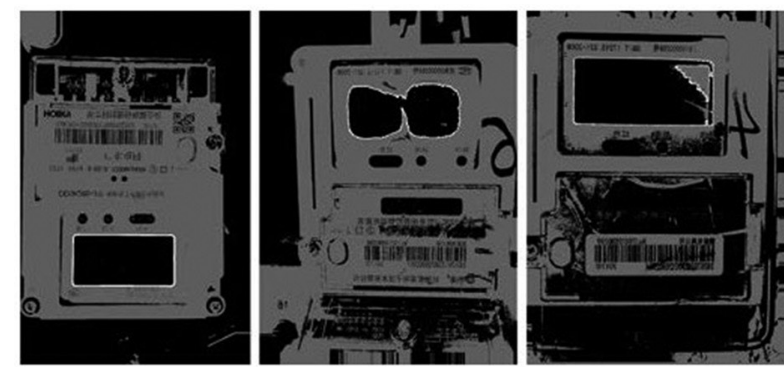

(b)
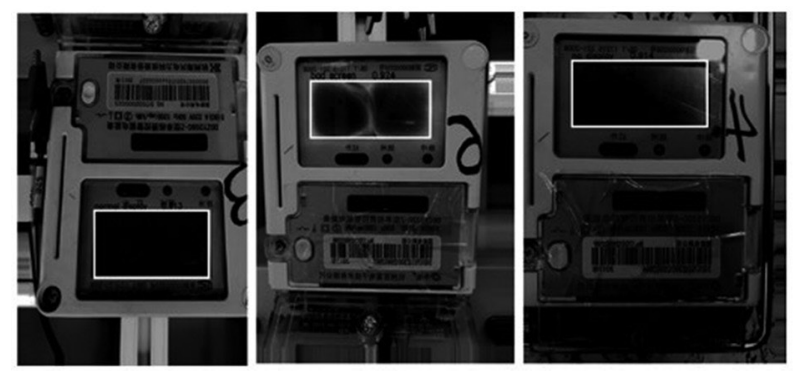

(c)
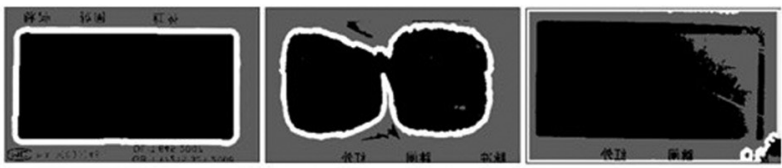

(d)

Figure 11. Samples of the basic global thresholding method and the YOLOv5.

\subsubsection{Evaluation of The Defect Detection Model}

At present, the LCD defect detection models of smart meters mainly use the template matching method, which can effectively detect whether the LCD screen normally displays. However, the template matching method can only detect and localize the LCD screen with incomplete display defects. Still, it cannot accurately identify the LCD screen with no display, liquid 
crystal rupture, abnormal display, and other defects.

We test the LCD defect detection model based on the Resnet34 on the LCD screen defect detection dataset. The experimental results show that the detection accuracy of our model exceeded 0.981 , and the detection accuracy of the five types of the LCD screen is shown in Table 1. For the classification of the types such as no display, abnormal display, liquid crystal display, and incomplete display, the model proposed by us has achieved satisfactory accuracy.

Table 2 is the confusion matrix of the prediction results of the five fault types of smart electricity meters. The model predicted the three samples, initially of no display type, to be of abnormal display type. The category of no display and the category of abnormal display have very similar features as shown in Figure 4, but they are two completely different states of the LCD screen. The features of the other categories have apparent differences and, therefore, the model can classify them accurately.

\subsubsection{Evaluation of The Overall Model}

We combine the trained LCD screen localization model with the defect detection model and test the performance of the two-stage model. The test result is shown in Table 3. The detection result samples of the overall model are as shown in Figure 12.

Table 1. The experimental results for five types of LCD screen.

\begin{tabular}{|c||c|c|c|c|c|c|c|}
\hline GT Predict & TP & FP & FN & TN & Precision & Recall & Accuracy \\
\hline \hline normal display & 64 & 0 & 0 & 102 & 1 & 1 & 1 \\
\hline no display & 35 & 0 & 3 & 128 & 1 & 0.9211 & 0.9819 \\
\hline abnormal display & 35 & 3 & 0 & 128 & 0.9211 & 1 & 0.9819 \\
\hline liquid crystal rupture & 18 & 0 & 0 & 148 & 1 & 1 & 1 \\
\hline incomplete display & 11 & 0 & 0 & 155 & 1 & 1 & 1 \\
\hline Average & & & & & 0.9842 & 0.9842 & 0.9928 \\
\hline
\end{tabular}

Table 2. Confusion matrix of experimental results for five types of LCD screens.

\begin{tabular}{|c||c|c|c|c|c|c|}
\hline GT Predict & $\begin{array}{c}\text { normal } \\
\text { display }\end{array}$ & no display & $\begin{array}{c}\text { abnormal } \\
\text { display }\end{array}$ & $\begin{array}{c}\text { liquid } \\
\text { crystal } \\
\text { rupture }\end{array}$ & $\begin{array}{c}\text { incomplete } \\
\text { display }\end{array}$ & Total \\
\hline \hline $\begin{array}{c}\text { normal } \\
\text { display }\end{array}$ & 64 & 0 & 0 & 0 & 0 & 64 \\
\hline no display & 0 & 35 & 3 & 0 & 0 & 38 \\
\hline $\begin{array}{c}\text { abnormal } \\
\text { display }\end{array}$ & 0 & 0 & 35 & 0 & 0 & 35 \\
\hline $\begin{array}{c}\text { liquid } \\
\text { crystal } \\
\text { rupture }\end{array}$ & 0 & 0 & 0 & 18 & 0 & 18 \\
\hline $\begin{array}{c}\text { incomplete } \\
\text { display }\end{array}$ & 0 & 0 & 0 & 0 & 11 & 11 \\
\hline \multicolumn{1}{c|}{ Total } & 64 & 35 & 38 & 18 & 11 & 166 \\
\hline
\end{tabular}


Table 3. The detection results of overall model and LSD+CRNN.

\begin{tabular}{|c|c|c|}
\hline Method & Type & Accuracy (\%) \\
\hline \hline LSD+CRNN[14] & $\begin{array}{c}\text { Location } \\
+ \\
\text { Detection }\end{array}$ & 95.7 \\
\hline Ours & $\begin{array}{c}\text { Location } \\
+ \\
\text { Detection }\end{array}$ & 98.9 \\
\hline
\end{tabular}

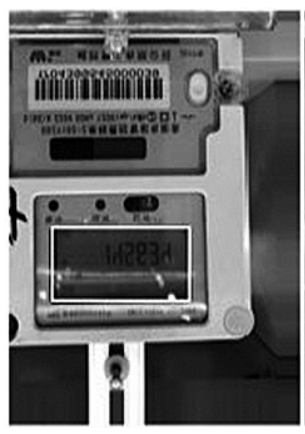

(a)

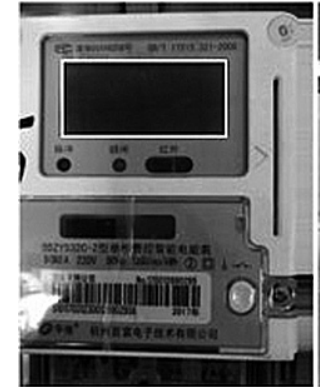

(c)

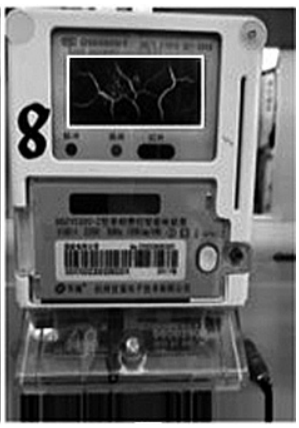

(b)

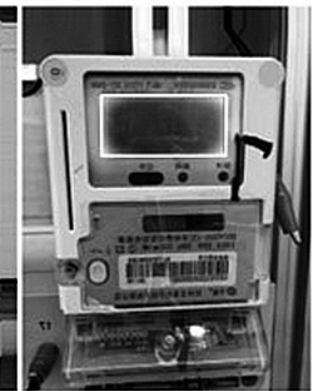

(d)
Figure 12. The detection result samples of the overall model.

Our two-stage defect detection model based on deep learning has achieved 98.9\% accuracy, which is better than the current defect detection model based on straight line detection.

\section{Conclusion}

Combining the YOLOv5 and ResNet34 deep learning models, we build a two-stage LCD screen defect detection model for smart meters. In addition, we constructed the LCD screen localization dataset and the LCD screen display defect dataset for the model training and testing.
The experimental results show that the LCD screen localization model we proposed has better performance than the basic global threshold method [2]. We proposed the LCD screen defect detection model to detect and classify the LCD screen display defects more comprehensively. The overall accuracy of our two-stage model reached $98.9 \%$. Compared with the state-ofthe-art method [14], our model achieves better performance. On the other hand, our model still has the potential to improve the accuracy of defect detection. The model has unsatisfactory accuracy for fault identification of incomplete LCD screen display. The model does not have character recognition ability in case a specific region of character is entirely missing.

Therefore, in future work, we plan to refer to the text recognition research to improve the model's character recognition ability to further enhance the performance of the LCD screen defect detection model.

\section{References}

[1] W. Wang, "Study on the Site Operation Management Strategy of the Smart Electricity Meter", Electrical measurement and instrumentation, vol. 51, no. 011, pp. 23-27, 2014. (in Chinese) http://dx.chinadoi.cn/10.3969/j.issn.1001-1390.2 014.11.006

[2] R. Chunlei, "Research of The Energy Meter Detection System Based on Machine Vision", Zhejiang University of technology, 2013. (in Chinese) https://doi.org/10.7666/d.Y2411250

[3] R. Gioi et al., "LSD: A Line Segment Detector", Image Processing On Line, vol. 2, no. 4, pp. 35-55, 2012.

https://doi.org/10.5201/ipol.2012.gjmr-lsd

[4] R. Gioi et al., "LSD: A Fast Line Segment Detector with a False Detection Control", IEEE Transactions on Pattern Analysis and Machine Intelligence, vol. 32, no. 4, 2010.

https://doi.org/10.1109/TPAMI.2008.300

[5] J. Illingworth and J. Kittler, "The Adaptive Hough Transform", IEEE Transactions on Pattern Analysis and Machine Intelligence, vol. 5, pp. 690-698, 1987.

https://doi.org/10.1109/TPAMI.1987.4767964

[6] S. Kang, "Research on Flat Panel Screen Detection Technology Based on Machine Vision", Shanghai Normal University, 2014. (in Chinese) http://dx.chinadoi.cn/10.7666/d.Y2526745 
[7] Z. Zhao, "Automatic Detection of Smart Meter Display Defects Based on Machine Vision", North China Electric Power University, 2018. (in Chinese) https://doi.org/10.7666/d.Y2526745

[8] W. Y. Qiao et al., "Research on Smart Meter Appearance Detection Based on Unified LBP Algorithm", in Proc. of the 2016 International Conference on Smart Grid and Electrical Automation (ICSGEA), 2016.

https://doi.org/10.1109/ICSGEA.2016.12

[9] R. Song et al., "A Novel Quality Test Method of Electricity Meter LCD Screen Based on Image Processing", Journal of Physics: Conference Series, 2018. https://doi.org/10.1088/1742-6596/1074/1/012167

[10] A. Anis et al., "Digital Electric Meter Reading Recognition Based on Horizontal and Vertical Binary Pattern", in Proc. of the 3rd International Conference on Electrical Information and Communication Technology (EICT), 2017. https://doi.org/10.1109/EICT.2017.8275241

[11] D. Quintanilha et al., "Automatic Consumption Reading on Electromechanical Meters Using HoG and SVM", in Proc. of the 7th Latin American Conference on Networked and Electronic Media (LACNEM 2017), 2017. http://doi.org/10.1049/ic.2017.0036

[12] W. Shujing and C. Kai, "Automatic Testing System Design of Smart Meter Based on Computer Vision", Electrical Measurement \& Instrumentation, vol. 53, no. 4, pp. 63-68, 2016. (in Chinese) https://doi.org/10.3969/j.issn.1001-1390.2016.04.011

[13] P. Yu, "Design of LCD Defect Detection System Based on Convolution Neural Network", Zhengzhou University, 2019, (in Chinese) [Online]. Available: https://kreader.cnki.net/Kreader/RedriectPage. aspx ?dbCode $=$ cdmd \& filename $=1019109138$. nh\&tablename $=$ CMFD201902

[14] B. Pei et al., "A Method of Detecting Defects of Smart Meter LCD Screen Based on LSD and Deep Learning", Multimedia Tools and Applications, pp. 1-18, 2021. http://doi.org/10.1007/s11042-020-10481-9

[15] G. Salomon et al., "Deep Learning for Image-Based Automatic Dial Meter Reading: Dataset and Baselines", in Proc. of the 2020 International Joint Conference on Neural Networks (IJCNN), 2020, pp. 1-8.

https://doi.org/10.1109/IJCNN48605.2020.9207318

[16] R. Girshick, "Fast r-cnn", in Proc. of the IEEE International Conference on Computer Vision, 2015, pp. 1440-1448.

http://doi.org/10.1109/ICCV.2015.169

[17] J. Redmon et al., "You Only Look Once: Unified, Real-Time Object Detection", in Proc. of the
IEEE Conference on Computer Vision and Pattern Recognition, 2016, pp. 779-788.

https://doi.org/10.1109/CVPR.2016.91

[18] J. Redmon and A. Farhadi, "YOLO9000: Better, Faster, Stronger", in Proc. of the IEEE Conference on Computer Vision and Pattern Recognition, 2017, pp. 7263-7271.

https://doi.org/10.1109/CVPR.2017.690

[19] J. Redmon and A. Farhadi, "Yolov3: An Incremental Improvement", arXiv preprint arXiv:1804.02767, 2018 [Online]. Available: https://arxiv.org/abs/1804.02767

[20] A. Bochkovskiy et al., "Yolov4: Optimal Speed and Accuracy of Object Detection", arXiv preprint arXiv:2004.10934, 2020 [Online]. Available: https://arxiv.org/abs/2004.10934

[21] C. Y. Wang et al., "CSPNet: A New Backbone that Can Enhance Learning Capability of CNN" in Proc. of the IEEE/CVF Conference on Computer Vision and Pattern Recognition Workshops, 2020, pp. 390-391.

https://doi.org/10.1109/CVPRW50498.2020.00203

[22] H. Kaiming et al., "Spatial Pyramid Pooling in Deep Convolutional Networks for Visual Recognition", IEEE Transactions on Pattern Analysis \& Machine Intelligence, 2015.

https://doi.org/10.1109/tpami.2015.2389824

[23] T. Y. Lin et al., "Feature Pyramid Networks for Object Detection", in Proc. of the IEEE Conference on Computer Vision and Pattern Recognition, 2017, pp. 2117-2125. https://doi.org/10.1109/CVPR.2017.106

[24] S. Liu et al., "Path Aggregation Network for Instance Segmentation", in Proc. of the IEEE Conference on Computer Vision and Pattern Recognition, 2018, pp. 8759-8768. https://doi.org/10.1109/CVPR.2018.00913

[25] H. Rezatofighi et al., "Generalized Intersection Over Union: A Metric and a Loss for Bounding Box Regression", in Proc. of the IEEE/CVF Conference on Computer Vision and Pattern Recognition, 2019, pp. 658-666. https://doi.org/10.1109/CVPR.2019.00075

[26] K. He et al., "Deep Residual Learning for Image Recognition", in Proc. of the IEEE Conference on Computer Vision and Pattern Recognition, 2016, pp. 770-778.

https://doi.org/10.1109/CVPR.2016.90 
Contact addresses:

Ye Chen

Electric Power Research Institute of Yunnan

Power Grid Co. Ltd

Yunnan Key Laboratory of Green Energy

Electric Power Measurement Digitalization

Control and Protection

China

e-mail: 363419628@qq.com

Zhihu Hong

Electric Power Research Institute of Yunnan

Power Grid Co. Ltd

China

e-mail: 842956127@qq.com

Yaohua Liao

Electric Power Research Institute of Yunnan

Power Grid Co. Ltd

Yunnan Key Laboratory of Green Energy

Electric Power Measurement Digitalization

Control and Protection

China

e-mail: 121051138@qq.com

Mengmeng Zhu

Electric Power Research Institute of Yunnan

Power Grid Co. Ltd

China

e-mail: 396923800@qq.com

Tong Han

Electric Power Research Institute of Yunnan

Power Grid Co. Ltd

Yunnan Key Laboratory of Green Energy

Electric Power Measurement Digitalization

Control and Protection

China

e-mail: 66097023@qq.com

Quan Shen

Beijing University of Posts and Telecommunications

Beijing

China

e-mail: 13051016253@163.com
Ye CHEN received master's degree in Power systems and automation from Kunming University of Science and Technology in 2017. His professional interests include topics in electric energy, electrical measurement and thermal engineering. He is a member of the High-precision Electrical Parameter Laboratory, Spark Power Research Studio, Intelligent Perception Innovation Studio, and Key Laboratory of CSG for Electric Power Measurement.

ZHIHU HoNG received his master's degree in electrical engineering from the Southwest Jiaotong University in 2018, and currently works as a high voltage researcher at the Yunnan Electric Power Research Institute of China Southern Power Grid. His research interests include insulation and condition assessment of high voltage electrical equipment, multi physical field finite element simulation of high voltage electrical equipment.

LiaO YAOHUA works as master engineer at the Yunnan Power Grid Co. Ltd. Electric Power Research Institute, engaged in electric energy, electrical measurement, thermal engineering, and high voltage measurement professional work. He secializes at solving measurement-related field problems. He is a member of the High-precision Electrical Parameter Laboratory, Spark Power Research, and Intelligent Perception Innovation Studio. He participated in the drafting of the Q/CSG 1209013.22019 and Q/CSG 1209013.7-2019 corporate standards.

Mengmeng Zhu works at the Electric Power Research Institute of Yunnan Power Grid Co.Ltd, as an senior engineer. His research interests include electric energy metering device technology research and power transformer field verification, AC / DC electronic transformer field key test technology application and distribution network fault detection and protection control work.

TON HaN works at the Electric Power Research Institute of Yunnan Power Grid Co.Ltd, as a senior engineer and Deputy Director of South Network Metrology key Laboratory. His research achievements include the Yunnan power grid natural disaster prevention monitoring and early warning system and risk control system, Dachaoshan, Manwan power plant generator set PSS parameters setting and field test and others. He is a first prize recipient of the Yunnan power grid science and technology progress award. He is long-termly engaged in grid relay protection technology, grid safety technology, measurement technology research work.

QuAN SHEN received his BSc degree in School of Automation, Beijing University of Posts and Telecommunications, Beijing, China, in 2016, where he is currently pursuing the master's degree in control science and engineering with the School of Automation. 